\title{
Front Matter: Volume 9330
}

, "Front Matter: Volume 9330," Proc. SPIE 9330, Three-Dimensional and Multidimensional Microscopy: Image Acquisition and Processing XXII, 933001 (20 April 2015); doi: 10.1117/12.2192399

SPIE. Event: SPIE BiOS, 2015, San Francisco, California, United States 


\section{PROGRESS IN BIOMEDICAL OPTICS AND IMAGING

\section{Three-Dimensional and Multidimensional Microscopy: Image Acquisition and Processing XXII}

Thomas G. Brown

Carol J. Cogswell

Tony Wilson

Editors

9-12 February 2015

San Francisco, California, United States

Sponsored and Published by

SPIE

Volume 9330 
The papers included in this volume were part of the technical conference cited on the cover and title page. Papers were selected and subject to review by the editors and conference program committee. Some conference presentations may not be available for publication. The papers published in these proceedings reflect the work and thoughts of the authors and are published herein as submitted. The publisher is not responsible for the validity of the information or for any outcomes resulting from reliance thereon.

Please use the following format to cite material from this book:

Author(s), "Title of Paper," in Three-Dimensional and Multidimensional Microscopy: Image Acquisition and Processing XXII, edited by Thomas G. Brown, Carol J. Cogswell, Tony Wilson, Proceedings of SPIE Vol. 9330 (SPIE, Bellingham, WA, 2015) Article CID Number.

ISSN: 1605-7422

ISBN: 9781628414202

Published by

SPIE

P.O. Box 10, Bellingham, Washington 98227-0010 USA

Telephone +1 3606763290 (Pacific Time) · Fax +1 3606471445

SPIE.org

Copyright (C) 2015, Society of Photo-Optical Instrumentation Engineers.

Copying of material in this book for internal or personal use, or for the internal or personal use of specific clients, beyond the fair use provisions granted by the U.S. Copyright Law is authorized by SPIE subject to payment of copying fees. The Transactional Reporting Service base fee for this volume is $\$ 18.00$ per article (or portion thereof), which should be paid directly to the Copyright Clearance Center (CCC), 222 Rosewood Drive, Danvers, MA 01923. Payment may also be made electronically through CCC Online at copyright.com. Other copying for republication, resale, advertising or promotion, or any form of systematic or multiple reproduction of any material in this book is prohibited except with permission in writing from the publisher. The CCC fee code is $1605-7422 / 15 / \$ 18.00$.

Printed in the United States of America.

Publication of record for individual papers is online in the SPIE Digital Library.

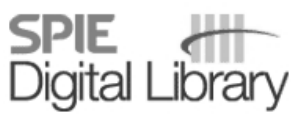

SPIEDigitallibrary.org

Paper Numbering: Proceedings of SPIE follow an e-First publication model, with papers published first online and then in print. Papers are published as they are submitted and meet publication criteria. A unique citation identifier (CID) number is assigned to each article at the time of the first publication. Utilization of CIDs allows articles to be fully citable as soon as they are published online, and connects the same identifier to all online, print, and electronic versions of the publication. SPIE uses a six-digit CID article numbering system in which:

- The first four digits correspond to the SPIE volume number.

- The last two digits indicate publication order within the volume using a Base 36 numbering

system employing both numerals and letters. These two-number sets start with 00, 01, 02, 03, 04, $05,06,07,08,09,0 A, 0 B \ldots 0 Z$, followed by 10-1Z, 20-2Z, etc.

The CID Number appears on each page of the manuscript. The complete citation is used on the first page, and an abbreviated version on subsequent pages. 


\title{
Contents
}

\author{
vii Authors \\ ix Conference Committee
}

INSTRUMENTAL METHODS: COHERENCE AND ILLUMINATION

933004 Development of cellular resolution Gabor-domain optical coherence microscopy for biomedical applications [9330-3]

933006 High brightness LED in confocal microscopy [9330-5]

933007 Limited-angle holographic tomography with optically controlled projection generation [9330-6]

INNOVATIVE INSTRUMENTAL METHODS

933008 Development of a DMD-based fluorescence microscope [9330-7]

933009 Multi-channel beam-scanning imaging at $\mathrm{kHz}$ frame rates by Lissajous trajectory microscopy [9330-8]

$9330 \mathrm{OA}$ Synchronous-digitization for video rate polarization modulated beam scanning second harmonic generation microscopy [9330-9]

INNOVATIVE MODES IN MULTIDIMENSIONAL MICROSCOPY

9330 OD A novel multimodal laser scanning microscope control system [9330-12]

$9330 \mathrm{OE} \quad$ Resolving power in direct oblique plane imaging [9330-13]

INNOVATIVE INSTRUMENTAL METHODS: 3D IMAGING

$9330003 \mathrm{D}$ microscope imaging robust to restoration artifacts introduced by optically thick specimens [9330-23]

$9330 \mathrm{OQ}$ Simultaneous multiplane imaging for 3D confocal microscopy using high-speed zscanning multiplexing [9330-25]

MULTIDIMENSIONAL IMAGE RECONSTRUCTION AND ANALYSIS

9330 OR Quantitative imaging of intact cardiac tissue using remote focusing microscopy [9330-27] 
9330 OS 3D widefield light microscope image reconstruction without dyes [9330-28]

9330 OT A block-based forward imaging model for improved sample volume representation in computational optical sectioning microscopy [9330-29]

9330 OU A robust simulation and reconstruction plafform of fluorescence molecular tomography [9330-30]

\section{COMPUTATIONAL MODELS}

9330 OV Computational modeling of STED microscopy through multiple biological cells under oneand two-photon excitation [9330-31]

9330 oW Fast calculation of the best focus position [9330-32]

9330 0X Nonlinear optical output of atomic systems via numerical simulation of time-dependent Schrodinger equations, stimulated by laser sources, in and out of resonance conditions within the non-perturbative regime [9330-33]

MULTIFOCAL METHODS AND EXTENDED DEPTH OF FOCUS

933010 Standing-wave excitation of fluorescence in a laser-scanning microscope allows precise contour mapping of the red blood cell membrane [9330-36]

933011 An information-theoretic approach to designing the plane spacing for multifocal plane microscopy [9330-37]

\section{QUANTITATIVE PHASE IMAGING}

933014 An integrated approach to determine prior information for improved wide-field imaging models from computational interference microscopy [9330-41]

933015 Analysis of dissected tissues with digital holographic microscopy: quantification of inflammation mediated tissue alteration, influence of sample preparation, and reliability of numerical autofocusing [9330-42]

933016 Dual-modality wide-field photothermal quantitative phase microscopy and depletion of cell populations [9330-43]

\section{COMPUTATIONAL METHODS}

933019 Dark-field illuminated fiber bundle endoscopy with iterative $\mathbf{1}_{1}$-min image reconstruction for honeycomb pattern removal [9330-46]

9330 1A Two-dimensional compressive sensing in spectral domain optical coherence tomography [9330-47] 
9330 1B Graphics processing unit-accelerated real-time compressive sensing spectral domain optical coherence tomography [9330-48]

\section{POSTER SESSION}

9330 ID Computed tomography of refractive index by low-coherence interferometry [9330-50]

$9330 \mathrm{lE} \quad$ A novel method for image denoising of fluorescence molecular imaging based on fuzzy C-Means clustering [9330-51]

9330 IG Optical coherence detection with stimulated emission: a feasibility study [9330-53]

$9330 \mathrm{1H} \quad$ High-resolution imaging of biological tissue with full-field optical coherence tomography [9330-54]

9330 1K A software tool for STED-AFM correlative super-resolution microscopy [9330-57]

$93301 \mathrm{M}$ Tomographic STED microscopy to study bone resorption [9330-59]

$93301 \mathrm{~N}$ Single-channel stereoscopic video imaging modality based on a transparent rotating deflector [9330-60]

933010 Resolution analysis in compressive multidimensional microscopy [9330-61]

9330 1Q Probing axial orientation of collagen fibers with Brillovin microspectroscopy [9330-63] 
Proc. of SPIE Vol. $9330933001-6$

Downloaded From: https://www.spiedigitallibrary.org/conference-proceedings-of-spie on 26 Apr 2023 Terms of Use: https://www.spiedigitallibrary.org/terms-of-use 


\title{
Authors
}

Numbers in the index correspond to the last two digits of the six-digit citation identifier (CID) article numbering system used in Proceedings of SPIE. The first four digits reflect the volume number. Base 36 numbering is employed for the last two digits and indicates the order of articles within the volume. Numbers start with 00, 01, 02, 03, 04, 05, 06, 07, 08, 09, 0A, 0B...0Z, followed by 10-1Z, 20-2Z, etc.

\author{
Abraham, Anish V., 11 \\ Amor, Rumelo, 10 \\ Amos, William Bradshaw, 10 \\ An, Yu, OU, 1E \\ Barnea, Itay, 16 \\ Belashenkov, Nikolai, OW \\ Bettenworth, Dominik, 15 \\ Bezzubik, Vitalii, OW \\ Blum, Omry, 16 \\ Bouman, Charles A., 09 \\ Brey, E., OS \\ Bub, G., OR \\ Burton, R. A. B., OR \\ Canavesi, Cristina, 04 \\ Chakrova, Nadya, 08 \\ Chao, Jerry, 11 \\ Chi, Chongwei, OU, IE \\ Chung, Shen-Shou Max, IG \\ Clemente, P., 10 \\ Corbett, A. D., OR \\ Davis, Mitchell A., OV \\ Deguchi, Takahiro, 1K, IM \\ DeWalt, Emma L., OA \\ Diaspro, Alberto, $\mathrm{OQ}$ \\ DiMarzio, Charles A., 06, OD, OX \\ Domagk, Dirk, 15 \\ Dunn, Andrew K., OV \\ Duocastella, Marti, OQ \\ Ezashi, T., OS \\ Gao, Wanrong, $1 \mathrm{H}$ \\ Ghosh, Sreya, OT \\ Gu, Zetong, OD \\ Ha, Myungjin, $1 \mathrm{~N}$ \\ Hänninen, Pekka E., 1K, $1 \mathrm{M}$ \\ Holmes, C., OS \\ Holmes, T., OS \\ Hossain, Md Shohag, 14 \\ Huang, Jinxin, 04 \\ Huang, Yong, 1A, 1B \\ Irles, E., 10 \\ Jang, Seul Gi, $1 N$ \\ Jiang, Shixin, OU, $1 \mathrm{E}$ \\ Jun, Eunkwon, $1 \mathrm{~N}$ \\ Jung, Byungjo, $1 \mathrm{~N}$ \\ Jurkevich, A., OS \\ Kang, Jin U., 1A, 1B \\ Kao, Fu-Jen, $1 G$ \\ Karasek, Stephen, OD, OX \\ Kemper, Björn, 15
}

\author{
Ketelhut, Steffi, 15 \\ Kim, Jeongmin, $\mathrm{OE}$ \\ King, Sharon V., 00, 14 \\ Kirby, Mitchell, 19 \\ Koho, Sami V., 1K, $1 \mathrm{M}$ \\ Korenstein, Rafi, 16 \\ Krausewitz, Philipp, 15 \\ Krauze, W., 07 \\ Kujawińska, M., 07 \\ Kuo, Wen-Chuan, $1 \mathrm{G}$ \\ Kuś, A., 07 \\ Lai, Zhenhua, OD \\ Lancis, J., 10 \\ Larkin, S., OS \\ Larson, J., OS \\ Lee, Sangyeob, $1 \mathrm{~N}$ \\ Lenz, Philipp, 15 \\ $\mathrm{Li}$, Tongcang, $\mathrm{OE}$ \\ Liu, Jie, OU, $1 \mathrm{E}$ \\ Liu, Xuan, 19 \\ Löhmus, Madis, IK \\ Ma, Zhenhe, ID \\ Mahajan, Sumeet, 10 \\ Mao, Yamin, OU, $1 \mathrm{E}$ \\ Mark, Andrew E., OV \\ McConnell, Gail, 10 \\ McLean, James, OD \\ Meng, Zhaokai, 1Q \\ Muir, Ryan D., 09, 0A \\ Näreoja, Tuomas, 1K, 1M \\ Newman, Justin A., 09 \\ Ober, Raimund J., 11 \\ Papavasiliou, G., OS \\ Park, Jihoon, $1 \mathrm{~N}$ \\ Patwary, Nurmohammed, 00 \\ Peltonen, Juha, 1M \\ Preza, Chrysanthe, 00, 0T, 14 \\ Qi, Shaohai, 19 \\ Radfar, Edalat, $1 \mathrm{~N}$ \\ Raj, Divyaansh, 19 \\ Rajadhyaksha, Milind, 06 \\ Ram, Sripad, 11 \\ Rieger, Bernd, 08 \\ Rodriguez, A. D., 10 \\ Rolland, Jannick P., 04 \\ Salvador, E., 10 \\ Santhanam, Anand P., 04 \\ Schmitt, Paul D., OA \\ Shaked, Natan T., 16
}


Simpson, Garth J., 09, 0A

Sinha, S., OS

Soldevila, F., 10

Sreehari, Suhas, 09

Stallinga, Sjoerd, 08

Starosta, Matthew S., OV

Sullivan, Shane Z., 09, OA

Tahmasbi, Amir, 11

Tajahuerce, E., 10

Tankam, Patrice, 04

Tian, Jie, OU, $1 \mathrm{E}$

Turko, Nir A., 16

Turturro, M., OS

Vaicik, M., OS

Vakili, Ali, 06

Vdovin, Gleb, OW

Vicidomini, Giuseppe, $0 Q$

Wang, Yi, ID

Wang, Yuan, OE

Ward, E. Sally, 11

Wilson, T., OR

Won, Jungeun, 04

Xiong, Daxi, 06, OD

$X U$, Daguang, 1A, 1B

Yakovlev, Vladislav V., 1Q

Yang, Xin, OU, IE

Ye, Jinzuo, OU, IE

Yin, Jihao, OD

Yu, Chun-hui, IG

Yu, Sung Kon, $1 \mathrm{~N}$

Zhang, Lijun, 19

Zhang, Xi, OD

Zhang, Xiang, OE

Zhao, Feng, 19

Zhou, Hongxian, 1D

Zhu, Yue, $1 \mathrm{H}$ 


\title{
Conference Committee
}

\author{
Symposium Chairs
}

James G. Fujimoto, Massachusetts Institute of Technology

(United States)

R. Rox Anderson, Wellman Center for Photomedicine, Massachusetts General Hospital (United States) and Harvard School of Medicine (United States)

Program Track Chairs

Ammasi Periasamy, University of Virginia (United States)

Daniel L. Farkas University of Southern California (United States)

Conference Chairs

Thomas G. Brown, University of Rochester (United States)

Carol J. Cogswell, University of Colorado at Boulder (United States)

Tony Wilson, University of Oxford (United Kingdom)

Conference Program Committee

Martin Booth, University of Oxford (United Kingdom)

G. J. Brakenhoff, Universiteit van Amsterdam (Netherlands)

José-Angel Conchello, Harvard University (United States)

Charles A. DiMarzio, Northeastern University (United States)

Raimund J. Ober, The University of Texas at Dallas (United States)

Chrysanthe Preza, University of Memphis (United States)

Monika Ritsch-Marte, Medizinische Universität Innsbruck (Austria)

\section{Session Chairs}

1 Instrumental Methods: Coherence and Illumination

Thomas G. Brown, University of Rochester (United States)

2 Innovative Instrumental Methods

Carol J. Cogswell, University of Colorado at Boulder (United States)

3 Innovative Modes in Multidimensional Microscopy

Laura Waller, University of California, Berkeley (United States)

4 Instrumental Methods: Biology \& Medicine

Alexander Jesacher, Medizinische Universität Innsbruck (Austria) 
5 Innovative Instrumental Methods: 3D Imaging

Thomas G. Brown, University of Rochester (United States)

6 Multidimensional Image Reconstruction and Analysis

Katherine Creath, Optineering (United States)

7 Computational Models

Raimund J. Ober, The University of Texas at Dallas (United States)

8 Multifocal Methods and Extended Depth of Focus

Charles A. DiMarzio, Northeastern University (United States)

9 Quantitative Phase Imaging

Sharon V. King, The University of Memphis (United States)

10 Computational Methods

Thomas G. Brown, University of Rochester (United States) 